MILITARY TECHNICAL COLLEGE CAIRO - EGYPT

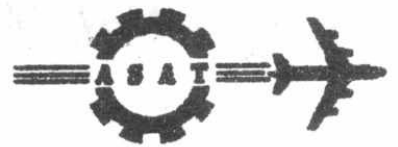

$7^{\text {th }}$ INTERNATIONAL CONF. ON AEROSPACE SCIENCES \& AVIATION TECHNOLOGY

\title{
OBSERVER DESIGN FOR LOOP TRANSFER RECOVERY
}

\author{
AHMED E. ABDALLA*
}

\begin{abstract}
A design technique of loop transfer recovery (LTR) is introduced to design a robust fullorder observer that recovers the full-state feedback loop properties for uniformly completely controllable (UCC) and uniformly completely observable (UCO) linear timevarying (LTV) systems. Asymptotic loop transfer recovery is achieved by selecting the observer gains based on the observability Gramian of closed loop system. We study the loop recovery problem at the input of the plant when the loop is broken at the input. When the loop is broken at the output, similar results can be developed for the recovery problem at the output of the plant. For linear time-invariant (LT) systems the observer gain is computed via solving a simpler algebraic equation compared with the standard linear quadratic Gaussian/loop transfer recovery (LQG/LTR). For linear time-varying (LTV) systems the stability of the closed loop system is guaranteed during the recovery process without any additional constrain. Finally, two examples are given to illustrate the effectiveness of the proposed approach.
\end{abstract}

\section{KEY WORDS}

Observability Gramian. Target Loop Dynamics, and Loop Transfer Recovery

\section{INTRODUCTION}

In model based controller design, when the plant model is known, the separation principle can be applied [1]. This means that the design can be performed in two separate steps. namely the state-feedback control design and observer design to reconstruct the state using only the output information. However, in the presence of model uncertainty, the controller design based on the separation principle does not necessarily have the same performance/robustness properties attainable by the state feedback design [2]. Thus, there is a definite need to develop observer design schemes

- Ph.D. Department Of Circuits and Systems. Military Technical College. Cairo, Egypt. 
which recover the properties achievable by the state-feedback design and, at the same time, guarantees the stability of the closed loop system. Over the past decade, loop transfer recovery (LTR) has been proposed as solution to the recovery of stability robustness and disturbance rejection characteristics in LQG [3-12]. The main idea in this design, is to select the observer gain such that a certain loop-transfer function is the same as in the state-feedback case [3]. This paper proposes a simple design rules of such observers for both LTI and LTV systems. The observer gain is a function of the observability Gramian of the closed loop system which is exponentially weighted by a control parameter. The system is assumed to be UCO, UCC, and minimum phase. We study the case when the loop is broken at the input point of the given system. However, when the loop is broken at the output point, a dual result can be developed. The degree to which the desirable target loop is recovered is partially dictated by the selection of the control weighting parameter. In Section 2, LTR design is made for completely observable LTI systems. The observer gain using the proposed LTR technique is computed via solving a simpler algebraic equation compared to the standard LQG/LTR where the computation involves the solution of Riccati equations [4]. In Section 3, we generalize the design. technique of the proposed LTR to the case of LTV systems. The stability of the closf $\mathrm{d}$ loop system is guaranteed during the recovery process without any additional constrain. While, applying the standard LQG/LTR for LTV systems required the solution of 1 frozen-time feedback algebraic Riccati equation. This approach assumes that the rate of the parameter variations is sufficiently small to guarantee the closed loop stability during the recovery process $[18,19]$. In Section 4 , we introduce two simple numerical examples to show the capability of the proposed technique.

\section{ASYMPTOTIC LTR OF LTI SYSTEMS}

Consider a LTI system

$$
\begin{aligned}
& \dot{x}(t)=A x(t)+B u(t) \\
& y(t)=C x(t)
\end{aligned}
$$

under the assumption of the pair $\{A, B\}$ is completely controllable and the pair $\{A, C\}$ is completely observable, we can assign the observability Gramian in observer design. Moreover the exponential rate of stability convergence and estimated error convergence can be controlled. This will be the key point in the designing of the loop transfer recovery. The observer deign are stated in the following theorem.

\section{Theorem 1}

The observer equation for the system given by (1) can be written as follows [1]:

$$
\dot{\hat{x}}(t)=F \hat{x}(t)-L y(t)+H u(t)
$$

where $\hat{x}(t)$ is the observer state vector, matrices $F$ and $H$ are chosen such that 
$F=A+L C, H=B$, and

$$
\mathrm{L}=-\mathrm{N}^{-1}(\delta) \mathrm{C}^{\mathrm{T}}
$$

$$
N(\delta)=\int_{0}^{0} e^{-\left(A^{T}+\beta I\right) \tau} C^{T} C e^{-(A+\beta 1) \tau} d \tau
$$

Let the error state vector be defined as

$$
e(t)=\hat{x}(t)-x(t)
$$

then the error dynamics described by the following differential equation

$$
\dot{e}(t)=(A+L C) e(t)
$$

is exponentially stable i.e. $(\mathrm{A}+\mathrm{LC})$ is Hurwitz.

\section{Proof}

Consider the associated scalar valued function

$$
\begin{aligned}
& v(e)=e^{T} N e \\
& \dot{v}(e)=e^{T}\left[(A+\beta I)^{T} N+N(A+\beta I)\right] e-2 e^{T} C^{T} C e-2 \beta e^{T} N e
\end{aligned}
$$

$N$ is positive definite by complete observability of the pair $\{A+\beta I, C\}$.

$$
\begin{aligned}
(A+\beta I)^{T} N+N(A+\beta I) & =-\int_{0}^{\delta} \frac{d}{d \tau}\left(e^{-\left(A^{T}+\beta I\right) \tau} C^{T} C e^{-(A+\beta I) \tau}\right) d \tau \\
& =-e^{-\left(A^{T}+\beta 1\right) \delta} C^{T} C e^{-(A+\beta 1) \delta}+C^{T} C \\
\dot{V}(e) & =-e^{T}\left(e^{-\left(A^{T}+\beta I\right) \delta} C^{T} C e^{-(A+\beta I) \delta}+C^{T} C\right) e-2 \beta e^{T} N e \\
& \leq-2 \beta e^{T} N e \\
& =-2 \beta V
\end{aligned}
$$

which implies that the error state exponentially goes to zero with rate $\beta$.

The case where $\beta=0 \quad\left\{N(\delta)=\int_{0}^{\delta} e^{-A^{r} t} C^{\top} C e^{-A \tau} d \tau\right\}$ is dual problem equivalent for the case proved in [14]. 


\subsection{Target Loop Dynamics}

Consider the block diagram shown in Figure 1 with loop broken at the indicated point, the open loop transfer function is given by

$$
G_{t}(s)=-K \Phi(s) B
$$

where $\Phi(s)=(s I-A)^{-1}$

\subsection{Recovery of The Target Loop Dynamics Via Model Based Compensator}

Consider the block diagram shown in Figure 2 with loop broken at the indicated point, the open loop transfer function is given by

$$
G_{r}(s)=K(s I-A-B K-L C)^{-1} L C \Phi(s) B
$$

From Theorem 1 and the separation design property, the stability of the closed system is guaranteed for all values of $\beta$. The proposed LTR gains have asymptotic properties which result in:

$$
\lim _{\beta \rightarrow \infty} G_{r}(s)=G_{t}(s)
$$

The asymptotic properties of the proposed LTR can be obtained by analyzing behavior of (8) as $\beta \rightarrow \infty$. It is loosely speaking as $\beta \rightarrow \infty$

$$
\lim _{\beta \rightarrow \infty} \frac{1}{2 \beta} L C=I
$$

where

$$
L=-N^{-1}(\delta) C^{r}
$$

Equation (11) can be written as follows

$$
G_{r}(s)=K\left[\frac{1}{2 \beta}(S I-A-B K)-\frac{1}{2 \beta}[C]^{-1} \frac{1}{2 \beta} L C \Phi(s) B\right.
$$

and from equation (13) we get the following

$$
\begin{aligned}
\lim _{\beta \rightarrow \infty} G_{r}(s) & =-K(s I-A)^{-i} B \\
& =G_{i}(s)
\end{aligned}
$$

It is clear from the previous equations that the control weighting parameter $\beta$ is the design variable for this procedure. The degree to which the desirable target loop 
recovered is partially established by the selection of the control weighting parameter $\beta$. The minimum phase restriction is obvious from equations (15) and (16) where the compensator poles will cancel the plant zeros and exact inversion over specified frequency range is possible.

\section{ASYMPTOTIC LTR OF LTV SYSTEMS}

Several approaches exist for studying the stability of the LTV systems. Depending on their framework, these studies can be classified as a state space approach and/or inputoutput approach $[1,15,16]$. The input-output description of a system gives a mathematical relation between the input and output of the system. In state-space approach, the system is described in terms of its internal behavior, referred to as states, and is governed by ordinary differential equations. A simple, but restrictive approach to investigate the stability of LTV systems is so-called frozen-time approach. In this approach, the system viewed as a collection of LTI systems for which well known LTI tools can be applied $[17,18]$. For example a sufficient condition for asymptotic stability of the LTV system is that the frozen system matrix is strictly Hurwitz and the rate of parameter variations is sufficiently small. In [19] the frozen-time approach is used for target loop recovery of time-varying systems. In general this approach dose not guarantee that the closed loop system will be stable during the recovery process. Thus, additional constrains should be imposed in odder to guarantee the stability. In this section we extend the proposed LTR to the case of LTV systems. The closed loop stability is guaranteed during the recovery. process without the need to additional constrains as in the case of the frozen-time approach.

\subsection{Observer Dynamics}

Consider the linear time-varying system described by

$$
\begin{aligned}
& \dot{x}(t)=A(t) x(t)+B(t) u(t) \\
& y(t)=C(t) x(t)
\end{aligned}
$$

where the pair $\{A(t), C(t)\}$ is UCO. The observer dynamics is described as follows

$$
\dot{\hat{x}}(t)=F(t) \hat{x}(t)-L(t) y(t)+H(t) u(t)
$$

where $\hat{x}(t)$ is the observer state vector. The matrices $F(t)$ and $H(t)$ are chosen such that

$$
F(t)=A(t)+L(t) C(t)
$$

where $H(t)=B(t), L(t)=-N^{-1}(t-\delta, t) C^{\top}(t)$, and $N(t-\delta, t)$ is the observability Gramian is defined by

$$
N(t-j, t)=\int_{t \rightarrow j}^{1} \Phi_{\beta}^{\Gamma}(\tau, t) C^{\top}(\tau) C(\tau) \Phi_{\beta}(\tau, t) d t
$$


where $\Phi_{\beta}(\tau, t)$ is the state transition matrix for $A(t)+\beta I$ with scalar parameter $\beta \geq 0$ and $\delta$ is the observability interval. It is straight forward to show that

$$
\Phi_{\beta}(\tau, \mathrm{t})=\mathrm{e}^{\beta(\tau-t)} \Phi(\tau, \mathrm{t}),
$$

where $\Phi(t, \tau)$ is the state transition matrix of LTV system in (17). An error state vector can be defined as $e(t)=\hat{x}(t)-x(t)$. Then the error dynamics can bè described as follows

$$
\dot{e}(t)=[A(t)+L(t) C(t)] e(t)
$$

where $e(t)=\hat{x}(t)-x(t)$

\section{Theorem 2}

The error state defined in (21) goes to zero exponentially fast with rate of at least $\beta$.

\section{Proof}

Consider the associated Scalar function

$$
V(e(t), t)=e^{\tau}(t) N(t-\delta, t) e(t)
$$

Since $N(t-\delta, t)$ is bounded, then we can obtain

$$
\alpha_{1}\|e(t)\|^{2} \leq V(e(t), t) \leq \alpha_{2}\|e(t)\|^{2}
$$

where $\alpha_{1} \& \alpha_{2}>0$

$$
\begin{aligned}
\frac{d}{d t} V(e(t), t) & =-e^{T}\left\{C^{\top} C(t)+\Phi_{\beta}^{\top}(t-\delta, t) C^{\top} C(t-\delta) \Phi_{\beta}(t-\delta, t)+2 \beta N(t-\delta, t)\right\} e(t) \\
& \leq-e^{T}\{2 \beta N(t-\delta, t)\} e(t) \\
& =-2 \beta V
\end{aligned}
$$

which implies that the error state exponentially goes to zero exponentially with rate $\beta$. The case where $\beta=0$ is a dual problem equivalent for the case proved in [20].

\subsection{Target Loop Dynamics}

Consider the block diagram shown in Figure 1 with loop broken at the input point, the open loop input/output relationship assuming zero initial conditions is given by

$$
u^{\prime}(t)=K(t) \int_{t_{0}}^{1} \Phi(t, \tau) B(\tau) u(\tau) d \tau
$$


where $\Phi(t, \tau)$ is the state transition matrix of the system in (17).

\subsection{Recovery of The Target Loop Dynamics Via Model Based Compensator}

Consider the block diagram shown in Figure 2 with loop broken at the input point, the open loop input/output relationship assuming zero. initial conditions is given by

$$
u^{\prime \prime}=-K(t) \int_{t_{0}}^{t} \Phi_{c}(t, \tau) L(\tau) C(\tau)\left[\int_{t_{n}}^{\tau} \Phi(\tau, s) B(s) u(s) d s\right] d \tau
$$

where $\Phi_{e}(t, \tau)$ is the state transition matrix of the following system

$$
\dot{\hat{x}}(t)=(A(t)+L(t) C(t)+B(t) K(t)) \hat{x}(t)
$$

From Theorem 2 the stability of the closed system is guaranteed for all values of $\beta$.

Define the following operator

$$
\Psi[r(t)]=\int_{t_{0}}^{t} \Phi(t, \tau) r(\tau) d \tau
$$

where $\Phi(\cdot$,$) is the state transition matrix of the LTV system \dot{x}(\mathrm{t})=\mathrm{A}(\mathrm{t}) \mathrm{x}(\mathrm{t})$. Equation (25) can be rewritten as follows

$$
u^{\prime \prime}(t)=-K\left(\Psi^{-1}-B K-L C\right)^{-1} L C \Psi[B u(t)]
$$

In the limit when $\beta \rightarrow \infty$

$$
\begin{aligned}
\lim _{\beta \rightarrow \infty} u^{\prime \prime} & =K \Psi[B u(t)] \\
& =u^{\prime}(t)
\end{aligned}
$$

Equations (29) and (30) requires that the system in (17) has to be exponentially stable zero dynamics.

\section{EXAMPLES AND SIMULATION RESULTS \\ Example 1 \\ Consider the following double-integrator plant}




$$
\begin{aligned}
& \dot{x}(t)=\left[\begin{array}{ll}
0 & 1 \\
0 & 0
\end{array}\right] x(t)+\left[\begin{array}{l}
0 \\
1
\end{array}\right] u \\
& y(t)=\left[\begin{array}{ll}
1 & 0
\end{array}\right] x(t)
\end{aligned}
$$

The plots for the closed-loop step response are shown in Figure 3. The open-loop Bode plots for the proposed LTR are shown in Figure 4 and Figure 5. It is clear that the step response of the recovered closed loop approaches the state feedback for increasing the values of $\beta$. Also, the frequency response magnitude and phase of the recovered loop approach that of the state feedback target loop or increasing the values of $\beta$.

\section{Example 2}

Consider the following LTV, UCC system

$$
\begin{aligned}
& \dot{x}(t)=\left[\begin{array}{ccc}
0 & 1 & 0 \\
0 & 0 & 1 \\
2 \sin t & 2 & 2 \cos t
\end{array}\right] x(t)+\left[\begin{array}{l}
0 \\
0 \\
1
\end{array}\right] u(t) \\
& y(t)=\left[\begin{array}{lll}
1 & 0 & 0
\end{array}\right] x(t)
\end{aligned}
$$

The step response of the closed loop system using different values of $\beta=(0,10,100,200)$ is shown in Figure 6 . Note that the step response approaches the state feedback case for increasing values of $\beta$.

\section{5-CONCLUSIONS}

Asymptotic loop transfer recovery is achieved by selecting the observer gains based on the exponentially-weighted observability Gramian of the closed loop system. The system is assumed to be UCC, UCO, and minimum phase. We study the case when the loop is broken at the input point of the given system. By the selection of the control weighting parameter we control the desirable degree to which the target loop is recovered. the convergence results are presented that are sufficient to ensure the validity of the proposed technique. For LTI system the observer gain is computed via solving a simple algebraic equation. The stability of the closed loop system is guaranteed during the recovery process without any additional constrain. Simple numerical examples are given to show the capability of the proposed technique.

\section{REFRENCES}

[1] Chen T.. Linear system theory and design. Holt, Rinehart and Winston. New York. (1984). 
[2] Doyle C. and Stein G., "Robustness with observers," IEEE Trans. on Automat. Contr., vol. Ac-24, no. 4, pp. 607-611, (1979).

[3] Athans M., "A tutorial on the LQG/LTR method," Proc. Amer. Contr. Conf., pp. 1289-1295, (1986).

[4] Stein G. and Athans M., "The LQG/LTR procedure for multivariable feedback control design," IEEE Trans. on Automat. Contr., vol..32, n0. 2, pp. 105-114, (1987).

[5] Rohrs C. E., Melsa J. L., and Schultz D. G., Linear control systems. McGrawHill, New York, (1993).

[6] Zhang Z. and Freudenberg J. S., "Loop transfer recovery for non-minimum phase plants," IEEE Trans. on Automat. Contr., vol. 35, no. 5, pp. 547-553, (1990).

[7] Saberi A. and Sannuti t P., "Observer design for loop transfer recovery and for uncertain dynamical systems," IEEE Trans. on Automat. Contr., vol. 35, no. 8, pp. 878-897, (1990).

[8] Calise A. J. and Prasad J. V. R., "Approximate loop transfer recovery method for designing fixed-order compensatros," J. Guidance, vol. 13, no. 2, pp. 297-302, (1990).

[9] Matson C. L. and Maybeck P. S., "On an assumed convergence result in the LQG/LTR technique," IEEE Trans. on Automat. Contr., vol. 36, no. 1, pp. 123-126, (1991).

[10] Monahemi M. M., Barlow J. B., and Leary D. P., "Design of reduced-order loop transfer recovery," J. of Guidance Control and Dynamics, vol. 15, no. 6, pp. 1320$1325,(1992)$.

[11] Imai H., "Simultaneous recovery of loop transfer property and disturbance attenuation property by high gain observer," Int. J. contr., vol. 57, no. 4, pp. 481852, (1993).

[12] Ray L. R., "Stability robustness of uncertain LQG/LTR systems," IEEE Trans. On. Automat. Contr., vol. 38, no. 2, pp. 304-308, (1993).

[13] Rachid A., "Comments on the loop transfer recovery," IEEE Trans. on Automat. Contr., vol. 40, no. 2, pp. 335-337, (1995).

[14] Kleinman David L., "An Easy Way To Stabilize A Linear Constant System," IEEE Trans. on Automat. Contr. pp. 692, (1970).

[15] Kailath T., Linear systems. Prentice-Hall, Englewood Cliffs, New Jersey, (1980)

[16] Haines L. H. and L. M. Sliverman, "Internal and external stability of the linear systems," J. of Math. Analys. Applicat., vol. 21, pp. 277-286,.(1968).

[17] Desoer C. A.. "Slowly varying system." IEEE Trans. on Automat. Contr., pp. 780781, (1969).

[18] Zhang J. F., "General lemmas for stability analysis of linear continuous-time systems with slowly time-varying parameters," Int. J. Control vol. 58, no. 6, pp. 1437-1444, (1993).

[19] Shamma J. S., Analysis and design of gain scheduled control systems. Ph. D. Dissertation. .VIT, Cambridge, MA. (1988).

[20] Abdalla E. Ahmed, Control of linear time-varying systems. Ph. D. Dissertation. ASU, Tempe. AZ, (1996). 


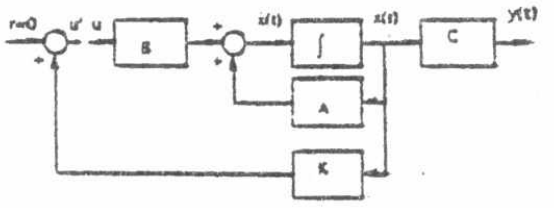

Figurer i: Target Loep Dyanica

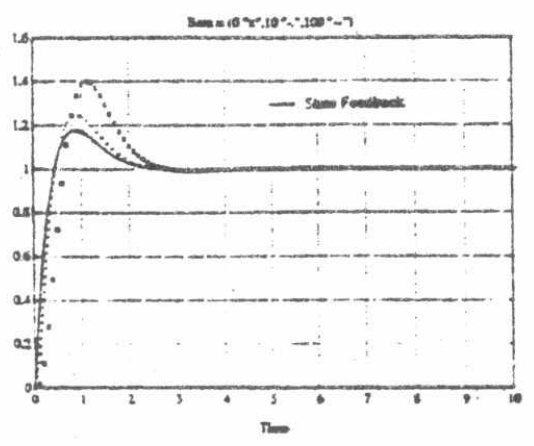

Figure 3. Step Reopones for the Propond LTR Usiag $\beta=(0,10,100)$

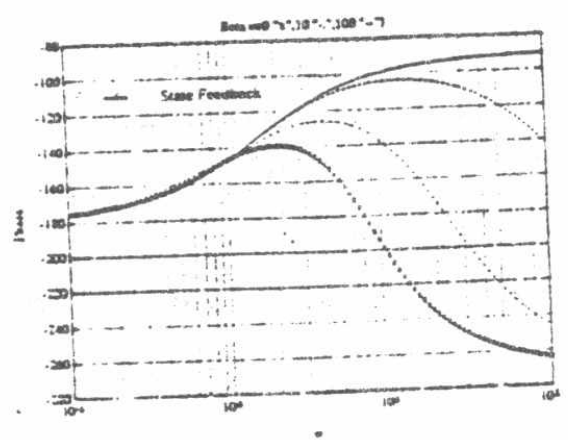

Figure 5: Open Loop Phase Bode Plots foc the Proposed LTR Uviag $\beta=(0,10,100)$

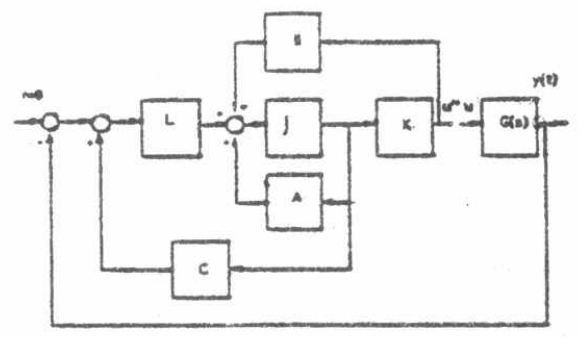

Figure 2: Recovery of Tarzes Loop Dynamica vis Moded Baed Compenemer

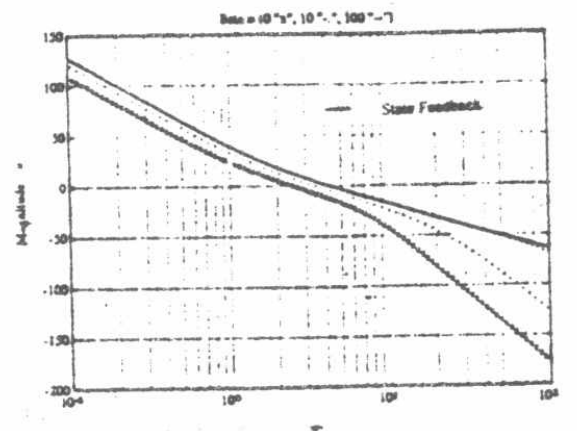

Figere t: Open Loop Magnitude Bode Ploce for the Proposed LTR Using $9=(0.10,100)$

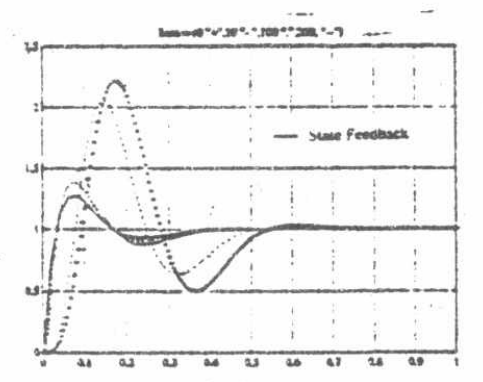

Fiture 6: Step Reuponse for the Proposed LTR for LTV Systems $B=(0.10 \cdot 100.200)$ 